\title{
Apatinib-treated advanced medullary thyroid carcinoma: a case report
}

This article was published in the following Dove Press journal:

OncoTargets and Therapy

\author{
Kan Chen \\ Yun Gao \\ Fei Shi \\ Guangqiang Cao \\ Jiandong Bao \\ Key Laboratory of Nuclear Medicine, \\ Ministry of Health, Jiangsu Key \\ Laboratory of Molecular Nuclear \\ Medicine, Jiangsu Institute of Nuclear \\ Medicine, Wuxi, China
}

\begin{abstract}
Medullary thyroid carcinoma (MTC) is a rare malignancy originating from calcitoninproducing parafollicular $\mathrm{C}$ cells of the thyroid. Neither radiotherapy nor chemotherapy has demonstrated durable objective responses in patients with advanced MTC. Vandetanib and cabozantinib are the 2 tyrosine kinase inhibitors recently approved by the US Food and Drug Administration, which are not affordable for most Chinese patients. Herein, we report a case of an MTC patient who responded to apatinib, a Chinese homemade tyrosine kinase inhibitortargeted vascular endothelial growth factor receptor. The patient was treated with thyroid lobectomy but developed MTC with extensive metastasis. The levels of serum calcitonin and carcino-embryonic antigen were much higher than the normal range. Apatinib was given at a dose of $500 \mathrm{mg}$ daily and adjusted according to tolerance. Sixteen weeks following apatinib administration, the patient achieved a partial response, which lasted more than 9 weeks. No severe toxicity or drug-related side effect was observed during the treatment. Therefore, apatinib could be a new option for the treatment of advanced MTC.
\end{abstract}

Keywords: apatinib, tyrosine kinase inhibitors, advanced medullary thyroid carcinoma

\section{Introduction}

Medullary thyroid carcinoma (MTC), a malignancy of the parafollicular C cells of the thyroid gland, accounts for $1 \%-2 \%$ of all thyroid cancers and presents either sporadically ( $75 \%$ of patients) or in a hereditary pattern. ${ }^{1}$ Central neck and lateral neck lymph node metastasis rates were $14 \%$ and $11 \%$ in patients with stage T1 MTC, and $86 \%$ and $93 \%$ in patients with stage T4, respectively. ${ }^{2}$ When MTC patients had nodules that could be touched, $70 \%$ of patients were found to have had cervical metastasis, and $10 \%$ of patients had distant metastases. ${ }^{3}$ The 10 -year survival rate of the patients with stage I, II, III, and IV MTC was $100 \%, 93 \%, 71 \%$, and $21 \%$, respectively. ${ }^{4}$ Neither radiotherapy nor chemotherapy has demonstrated durable objective responses in patients with advanced MTC. ${ }^{5,6}$

Germline mutations in the RET (rearranged during transfection) proto-oncogene occur in virtually all patients with hereditary MTC. ${ }^{7-9}$ Approximately $50 \%$ of the patients with sporadic MTC have somatic RET mutations.

In addition to RET, the hepatocyte growth factor receptor (MET) and vascular endothelial growth factor receptor 2 (VEGFR-2) signaling pathways are upregulated in thyroid tumors ${ }^{10,11}$ and implicated in the pathogenesis of MTC through promotion of proinvasive and proangiogenic phenotypes. ${ }^{12-14}$

Apatinib, which is a small molecule tyrosine kinase inhibitor (TKI), selectively inhibits VEGFR-2 and also moderately inhibits c-Kit, c-Src and RET. By binding to VEGFR-2, apatinib inhibits tyrosine kinase phosphorylation as well as its downstream 
signaling pathway, and then inhibits the proliferation and migration of vascular endothelial cells. ${ }^{15,16}$ Apatinib has been proven to be effective and safe in treating chemotherapyrefractory advanced gastric cancer and more commonly used for treating advanced breast cancer, soft tissue sarcoma, non-small cell lung cancer, and hepatocellular carcinoma. However, the efficacy of apatinib in MTC patients has not been reported yet. Herein, we first report a case of an MTC patient who responded to apatinib.

\section{Case report}

A 69-year-old man was hospitalized on May 16, 2016 because of MTC with extensive metastasis. He had 30 years history of smoking and drinking, and his mother had cardiac cancer. The patient's serum carcino-embryonic antigen (CEA) was elevated from the normal range to $10.4 \mathrm{ng} / \mathrm{mL}$ (normal value range is $0-7 \mathrm{ng} / \mathrm{mL}$ ) during health examination on August 15, 2006. He had undergone endoscopic resection of colon polyps several times. However, the serum CEA level further increased and reached $267 \mathrm{ng} / \mathrm{mL}$ on February 27, 2009. On August 14, 2009, a thyroid nodule was found and the patient underwent left thyroid lobectomy and right sub-total thyroidectomy. The postoperative pathology suggested bilateral multinodular goiter, and levothyroxine (Euthyrox, Merck KGaA, Darmstadt, Germany) $150 \mu \mathrm{g}$ qd was administrated. The patient did not suffer from hoarseness, choking, or limb twitching after surgery. The serum CEA level was $10.3 \mathrm{ng} / \mathrm{mL}$ on November 27, 2009, and then increased continually. On September 1, 2010, the patient's serum calcitonin level was $131 \mathrm{pg} / \mathrm{mL}$ (normal value range is $1-5.17 \mathrm{pg} / \mathrm{mL}$ ) and pathology confirmed a diagnosis of MTC. No abnormality was found during bone scan on December 6, 2010. Chest and abdominal CT scans on January 26, 2011 revealed focal liver lesions (possible malignancy). Robot-assisted partial right liver lobe resection was performed on March 16, 2011. The postoperative pathology revealed liver metastasis of MTC. CT scans in July 2012 revealed mediastinum and right hilar lymph node enlargement, possible double-pulmonary metastasis, multiple small cysts in the liver, double renal cyst, double kidney stones, and low-density shadow of the posterior segment of the right lobe of the liver. The target therapy sorafenib (400-600 mg qd) was given in August 2012. Sorafenib therapy failed to relieve the metastases in 1.5 years. On December 7, 2015, PET/CT examinations revealed multiple lung nodules and increased glucose metabolism after thyroid operation and liver metastasis operation, multiple low-density foci in the liver and increased glucose metabolism, bilateral adrenal local glucose metabolism increased and the right side twelfth rib local glucose metabolism increased, multiple enlarged lymph nodes with increased glucose metabolism in left subclavian, and right hilar, mediastinum, and hepatic hilar region. The results were suggestive of double lung, liver, bone, adrenal gland, and lymph node metastasis. The patient's serum thyroglobulin was $0.45-1.24 \mathrm{ng} / \mathrm{mL}$, serum CEA level was higher than 1,000 ng/mL since March 26, 2014, and even reached 8,757 ng/mL on April 6, 2016.

When the patient was admitted to the hospital, his physical examination results were good. Thyroid function-related parameters were in the normal range. The concentration of serum calcitonin was $67,420.00 \mathrm{pg} / \mathrm{mL}$ and CEA was $10,580.00 \mathrm{ng} / \mathrm{mL}$. CT scans revealed that both lungs had multiple metastases, mediastinal lymph node metastasis, multiple liver metastases, and hilar lymph nodes (Figure 1). After providing written informed consent, the patient received the treatment of apatinib, $500 \mathrm{mg} \mathrm{qd}$, and 3 days later, the dose was increased to $750 \mathrm{mg}$ qd. Levothyroxine $150 \mu \mathrm{g}$ qd was administrated simultaneously. Dose reduction of apatinib to $500 \mathrm{mg}$ qd occurred at week 4 because of hand-foot skin reaction (grade 2). Stable disease was observed during CT re-examination at week 8 (Figure 2 ). The concentrations
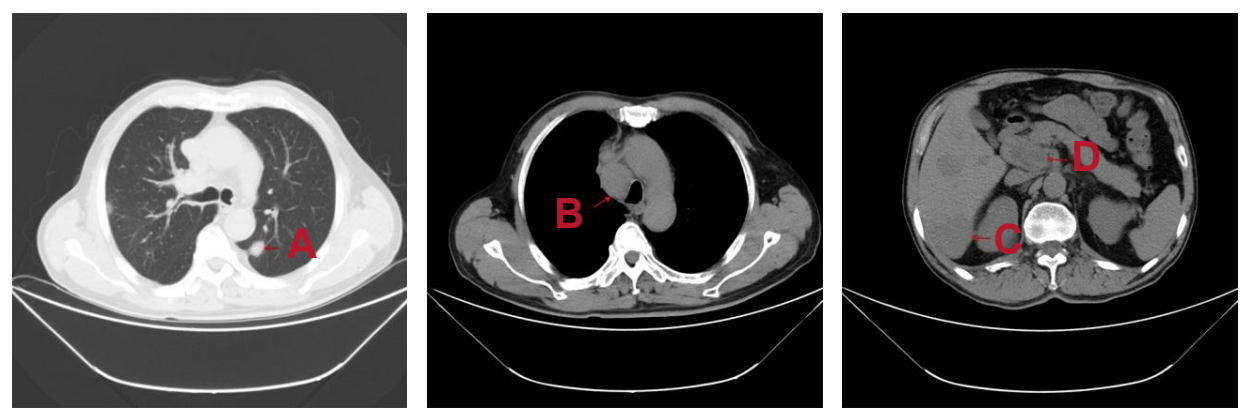

Figure I Advanced medullary thyroid carcinoma before treatment with apatinib.

Notes: (A) Both lungs with multiple metastases. (B) Mediastinal lymph node metastasis. (C) Multiple liver metastases. (D) Hilar lymph nodes. 

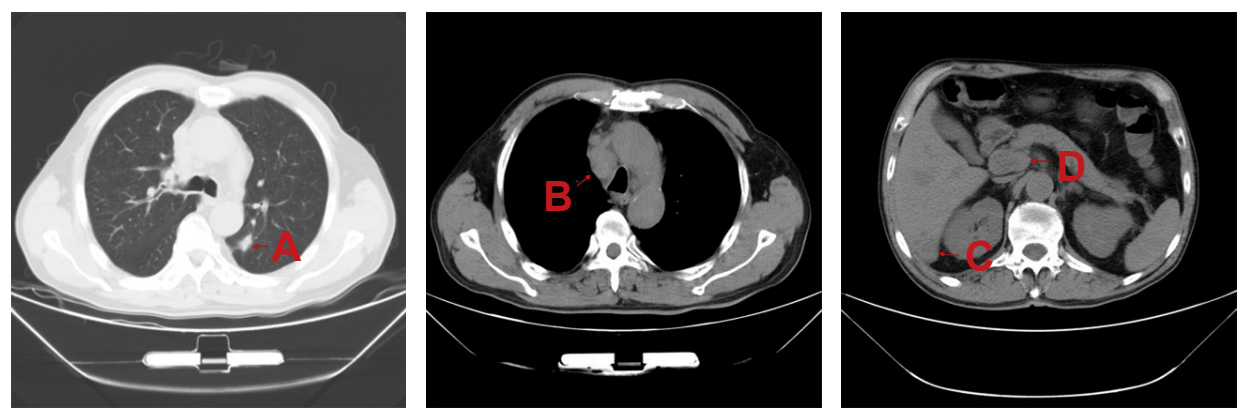

Figure 2 Advanced medullary thyroid carcinoma after 8 weeks of treatment with apatinib.

Notes: (A) Both lungs with multiple metastases. (B) Mediastinal lymph node metastasis. (C) Multiple liver metastases. (D) Hilar lymph nodes.

of serum calcitonin and CEA decreased dramatically $(16,670.00 \mathrm{pg} / \mathrm{mL}$ and 2,713.00 ng/mL, respectively). Partial response (PR) was observed at week 16 (Figure 3) and the concentrations of serum calcitonin and CEA continued to decline $(4,476.50 \mathrm{pg} / \mathrm{mL}$ and $1,865.00 \mathrm{ng} / \mathrm{mL}$, respectively). After 27 weeks of medication, apatinib administration was suspended for 2 weeks because of skin pain that the patient could not tolerate. Apatinib (250 mg qd) maintenance therapy lasted until week 40. The levels of serum calcitonin and CEA increased slightly, but the efficacy of PR was sustained.

The patient experienced some toxicities, but no serious adverse effects were observed. Hand-foot skin reaction occurred after 1 week of medication and aggravated to grade 2 after 3 weeks. Diarrhea and anorexia occurred after 14 weeks of medication and then aggravated too, which resulted in a 1-week period off treatment of apatinib. Apatinib $500 \mathrm{mg}$ /day was administrated again after symptomatic and supportive treatment of diarrhea. Urinary protein $(+)$ and occult blood of feces $(+)$ were controllable and tolerable. During the maintenance therapy of apatinib (250 mg qd), telmisartan (40 mg bid) was administrated for the treatment of hypertension.

PR was confirmed by CT scans after 25 weeks of treatment. By the end of February 2017, the administration of apatinib was continued with $250 \mathrm{mg} /$ day and the duration of treatment reached 40 weeks. Eastern Cooperative Oncology Group performance status of the patient was 1 .

Written informed consent was obtained from the patient for publication of this case report and accompanying images.

\section{Discussion}

Differentiated thyroid cancer (DTC), originating from thyroid follicular epithelium encompasses both papillary and follicular carcinoma, accounting for more than $90 \%$ of all thyroid cancers. ${ }^{17}$ After treatment with standard operation, radioactive iodine (RAI) treatment and thyroid-stimulating hormone suppressive therapy, most of the patients with DTC have good prognoses. ${ }^{18}$

MTC originates from thyroid follicular C cells, so the ability of tumor cells to absorb iodine is low, leading to poor treatment effect of RAI. MTC is one of the manifestations of multiple endocrine neoplasms in some patients. All these results suggest that MTC treatment is not effective and the prognosis is poor.

Vandetanib (2011) and cabozantinib (2012) have been approved for the treatment of advanced MTC. Vandetanib is an oral TKI that selectively targets RET, VEGFR, and epidermal growth factor receptor signaling. The efficacy
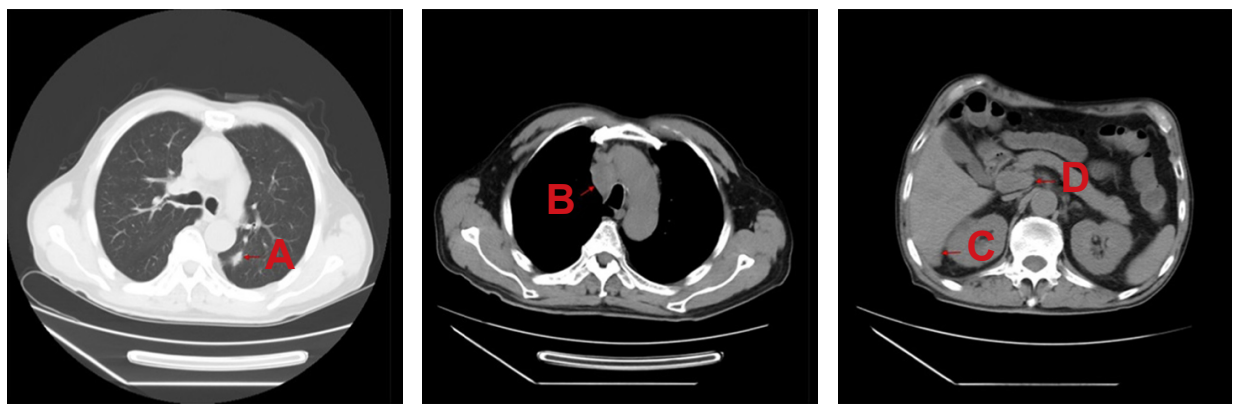

Figure 3 Advanced medullary thyroid carcinoma after 16 weeks of treatment with apatinib.

Notes: (A) Both lungs with multiple metastases. (B) Mediastinal lymph node metastasis. (C) Multiple liver metastases. (D) Hilar lymph nodes. 

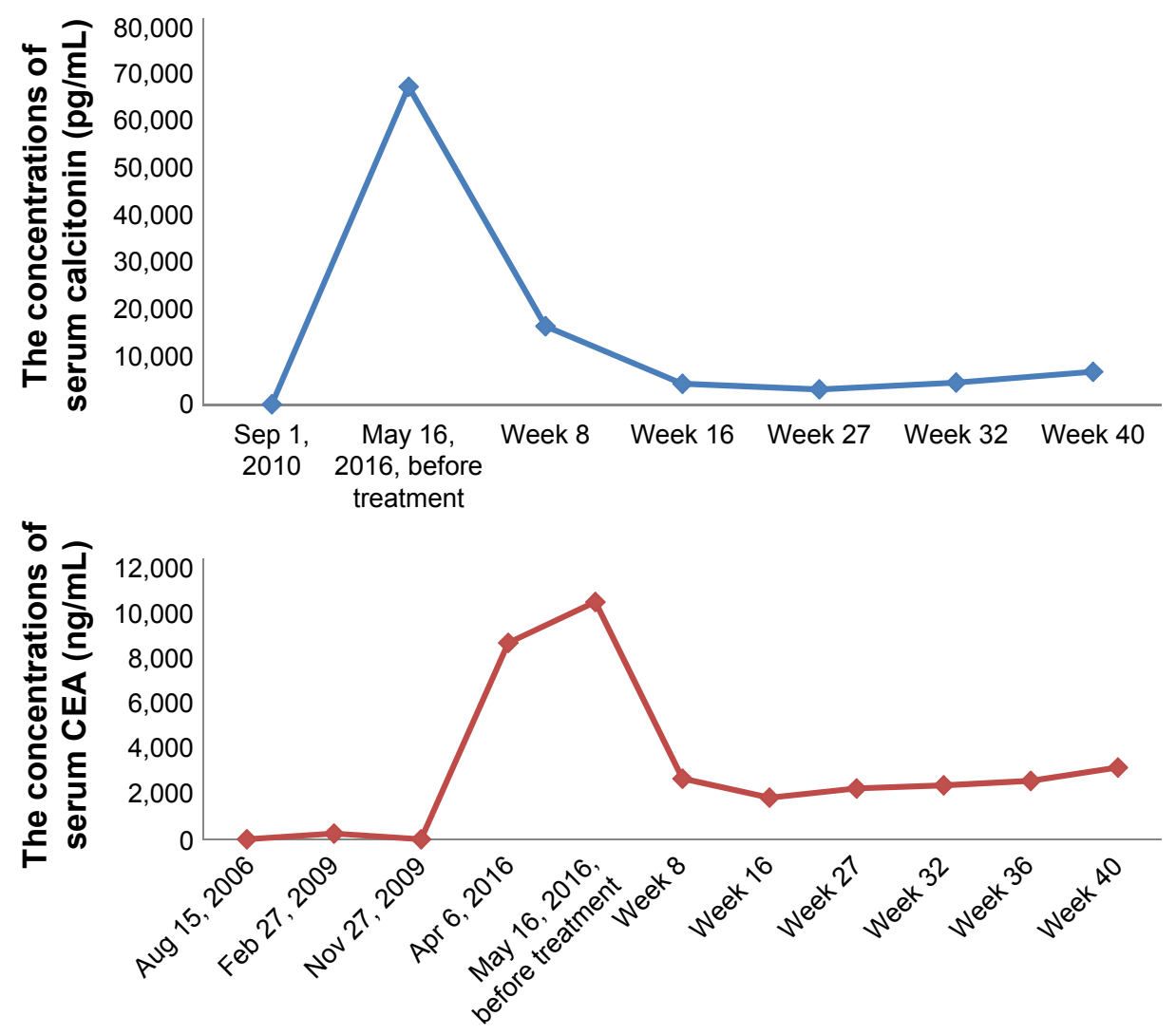

Figure 4 The levels of serum calcitonin and CEA continued to fall during treatment. Abbreviation: CEA, carcino-embryonic antigen.

of vandetanib in the treatment of MTC was evaluated in a Phase III trial. The study met its primary objective of progression-free survival (PFS) prolongation with vandetanib versus placebo (hazard ratio, $0.46 ; 95 \% \mathrm{CI}, 0.31$ to $0.69 ; P<0.001) .{ }^{19}$ Cabozantinib is a TKI that targets 3 relevant pathways in MTC: MET, VEGFR-2, and RET. In a Phase III study, cabozantinib demonstrated promising clinical activity with median PFS 11.2 months versus 4.0 months for placebo (hazard ratio, 0.28 ; 95\% CI, 0.19-0.40; $P<0.001){ }^{20}$

Angiogenesis plays an important role in tumor growth and metastasis. VEGFR is a critical target in antiangiogenesis treatment. Apatinib can selectively block VEGFR-2 and its downstream signaling pathway to inhibit tumor growth. ${ }^{15,16}$ It is reported that a safe and rapid response and high PR rate (referring to Response Evaluation Criteria in Solid Tumors 1.1) could be observed in RAI-refractory DTC patients within 8 weeks of apatinib treatment. ${ }^{21}$ In this case, we tried apatinib for the treatment of MTC with extensive metastasis. The preliminary results indicate that apatinib has outstanding efficacy for advanced MTC. The effect of PR lasted
25 weeks. Until the end of February 2017, the administration of apatinib was continued, and the estimated PFS was more than 9 months. Serum levels of calcitonin and CEA are important indicators of tumor burden and prognosis in MTC. From baseline to week 16, the patient displayed significant decreases in calcitonin and CEA (Figure 4).

The common adverse effects of apatinib included hand-foot skin reaction, high blood pressure, proteinuria, neutropenia, thrombocytopenia, throat pain, dysphagia, and diarrhea. In this case, the patient experienced hand-foot skin reactions and diarrhea. The adverse effects were controllable by dose reduction or interruption and symptomatic treatment.

In conclusion, apatinib is a drug with independent intellectual property rights. Its efficacy is clear and price is significantly lower than other molecular-targeted therapeutic drugs. Apatinib is a good option for the treatment of patients with advanced MTC in China.

\section{Disclosure}

The authors report no conflicts of interest in this work. 


\section{References}

1. Cancer of the Thyroid (Invasive): Trends in SEER Incidence and U.S. Mortality Using the Joinpoint Regression Program,1975-2014 With up to Five Joinpoints, 1992-2014 With up to Four Joinpoints, Both Sexes by Race/Ethnicity. Available from: https://seer.cancer.gov/csr/1975_2014/ results_merged/sect_26_thyroid.pdf\#search=cancer+of+the+thyroid+i nvasive. Accessed December 28, 2017.

2. Machens A, Hinze R, Thomusch O, Dralle H. Pattern of nodal metastasis for primary and reoperative thyroid cancer. World J Surg. 2002;26(1):22-28.

3. Moley JF. Medullary thyroid carcinoma: management of lymph node metastases. J Natl Compr Canc Netw. 2010;8(5):549-556.

4. Modigliani E, Cohen R, Campos JM, et al. Prognostic factors for survival and for biochemical cure in medullary thyroid carcinoma: results in 899 patients. The GETC Study Group. Groupe d'etude des tumeurs a calcitonine. Clin Endocrinol (Oxf). 1998;48(3):265-273.

5. Martins RG, Rajendran JG, Capell P, Byrd DR, Mankoff DA. Medullary thyroid cancer: options for systemic therapy of metastatic disease? J Clin Oncol. 2006;24(11):1653-1655.

6. American Thyroid Association Guidelines Task Force, Kloos RT, Eng C, et al. Medullary thyroid cancer: management guidelines of the American Thyroid Association. Thyroid. 2009;19(6):565-612.

7. Donis-Keller H, Dou S, Chi D, et al: Mutations in the RET protooncogene are associated with MEN 2A and FMTC. Hum Mol Genet. 1993;2(7):851-856.

8. Mulligan LM, Kwok JB, Healey CS, et al. Germline mutations of the RET proto-oncogene in multiple endocrine neoplasia type $2 \mathrm{~A}$. Nature. 1993;363(6428):458-460.

9. Carlson KM, Dou S, Chi D, et al. Single missense mutation in the tyrosine kinase catalytic domain of the RET protooncogene is associated with multiple endocrine neoplasia type 2B. Proc Natl Acad Sci US A. 1994;91(4):1579-1583.

10. Klein M, Picard E, Vignaud JM, et al. Vascular endothelial growth factor gene and protein: strong expression in thyroiditis and thyroid carcinoma. J Endocrinol. 1999;161(1):41-49.
11. Brose MS, Nutting CM, Jarzab B, et al; DECISION investigators. Sorafenib in radioactive iodine-refractory, locally advanced or metastatic differentiated thyroid cancer: a randomized, double-blind, phase 3 trial. Lancet. 2014;384(9940):319-328.

12. Papotti M, Olivero M, Volante M, et al. Expression of hepatocyte growth factor (HGF) and its receptor (MET) in medullary carcinoma of the thyroid. Endocr Pathol. 2000;11(1):19-30.

13. Soh EY, Duh QY, Sobhi SA, et al. Vascular endothelial growth factor expression is higher in differentiated thyroid cancer than in normal or benign thyroid. J Clin Endocrinol Metab. 1997;82(11):3741-3747.

14. Cassinelli G, Favini E, Degl'Innocenti D, et al. RET/PTC1-driven neoplastic transformation and proinvasive phenotype of human thyrocytes involve Met induction and beta-catenin nuclear translocation. Neoplasia. 2009;11(1):10-21.

15. Li J, Qin S, Xu J, et al. Apatinib for chemotherapy-refractory advanced metastatic gastric cancer: results from a randomized, placebo-controlled, parallel-arm, phase II trial. J Clin Oncol. 2013;31(26):3219-3225.

16. Li J, Qin S, Xu J, et al. Randomized, double-blind, placebo-controlled phase III trial of apatinib in patients with chemotherapy-refractory advanced or metastatic adenocarcinoma of the stomach or gastroesophageal junction. J Clin Oncol. 2016;34(13):1448-1454.

17. Sherman SI. Thyroid carcinoma. Lancet. 2003;361:501-511.

18. Haugen BR, Alexander EK, Bible KC, et al. 2015 American Thyroid Association Management Guidelines for Adult Patients with Thyroid Nodules and Differentiated Thyroid Cancer. Thyroid. 2016;26(1): $1-133$.

19. Wells SA Jr, Robinson BG, Gagel RF, et al. Vandetanib in patients with locally advanced or metastatic medullary thyroid cancer: a randomized, double-blind phase III trial. J Clin Oncol. 2012;30(2):134-141.

20. Elisei R, Schlumberger MJ, Müller SP, et al. Cabozantinib in progressive medullary thyroid cancer. J Clin Oncol. 2013;31(29):3639-3646.

21. Lin Y, Wang C, Li H, Liang J. The preliminary report about the efficacy and safety evaluation of apatinib in progressive radioactive iodinerefractory differentiated thyroid cancer within 8 weeks. China Oncol. 2016;26(9):721-726.
OncoTargets and Therapy

\section{Publish your work in this journal}

OncoTargets and Therapy is an international, peer-reviewed, open access journal focusing on the pathological basis of all cancers, potential targets for therapy and treatment protocols employed to improve the management of cancer patients. The journal also focuses on the impact of management programs and new therapeutic agents and protocols on

\section{Dovepress}

patient perspectives such as quality of life, adherence and satisfaction. The manuscript management system is completely online and includes a very quick and fair peer-review system, which is all easy to use. Visit http://www.dovepress.com/testimonials.php to read real quotes from published authors. 